'Escuela de Psicología, Universidad de los Andes. Santiago, Chile. ${ }^{2}$ Escuela de Enfermería (Campus San Felipe), Universidad de Valparaíso. San Felipe, Chile. ${ }^{3}$ Facultad de Educación, Pontificia Universidad Católica de Chile. Santiago, Chile.

${ }^{4}$ College of Nursing, Chang Gung University of Science and Technology. Taiwán aEnfermera. bsicólogo.

Recibido el 22 de junio de 2013 aceptado el 10 de abril de 2014

Correspondencia a: Dr. Jorge Gaete Monseñor Álvaro del Portillo No 12455, Las Condes. Chile. jgaete@uandes.cl.

\section{Factores asociados a conductas promotoras de salud en adolescentes chilenos}

\author{
JORGE GAETE ${ }^{1}$, ESTERBINA OLIVARES ${ }^{2, \mathrm{a}}$, \\ CRISTIAN A. ROJAS-BARAHONA ${ }^{3, b}$, NICOLÁS LABBÉ ${ }^{1, b}$, \\ MANUEL RENGIFO ${ }^{1, \mathrm{~b}}$, MAGDALENA SILVA ${ }^{2, \mathrm{a}}$, LETICIA LEPE ${ }^{2, \mathrm{a}}$, \\ CYNTHIA YÁÑEZ ${ }^{2, a}$, MEI-YEN CHEN ${ }^{4, a}$
}

\section{Factors associated with health promoting behaviors among Chilean adolescents}

Background: Health-promoting behaviors are important to prevent diseases and prolong life in the population. People develop these behaviors throughout life. However, better benefits for health are obtained with an early development. Aim: To determine the prevalence of health-promoting behaviors among early adolescents and its associated factors. Material and Methods: Cross-sectional survey performed in 1,465 students of high, medium and low socio-economic status, attending fifth to eighth grades of schools located in a small Chilean city. Participants answered a questionnaire that gathered information about frequency of health-promoting behaviors such as health responsibility and nutrition, physical exercise and stress management, life appreciation, social support and different personal, school and familial factors. Results: A higher frequency of health-promoting behaviors was associated with better academic achievement, better school commitment, and higher perception of school membership. It also was associated with a better perception of health status and a higher conformity with physical appearance. Conclusions: Health promoting behaviors in these children are related to a better academic achievement and a higher integration with school environment.

(Rev Med Chile 2014; 142: 418-427)

Key words: Adolescents; Health-behavior; Risk factors.
$\mathrm{E}$ 1 desarrollo de conductas promotoras de salud es uno de los grandes desafíos propuestos por la Organización Mundial de la Salud $(\mathrm{OMS})^{1}$. Estas conductas promueven el bienestar y desarrollo de la persona $a^{2,3}$ y disminuyen la aparición de patologías que comprometen la vida, tales como las enfermedades crónicas no transmisibles (ECNTs) ${ }^{3-5}$.

Las conductas promotoras en salud se adquieren tempranamente, pero durante la adolescencia también aparecen una serie de prácticas que podrían poner en riesgo la salud ${ }^{6}$. Por ejemplo, en Chile 25,9\% de los estudiantes entre octavo básico y cuarto año de enseñanza media refieren haber consumido algún cigarrillo durante el último mes y las cifras de consumo de alcohol y marihuana ascienden a $34,7 \%$, y $11,2 \%$, respectivamente ${ }^{7}$. Por otro lado, la Encuesta Nacional de Salud (2009$2010)^{9}$ muestra que en la población entre 15 y 24 años, el sobrepeso llega a 27\%, la obesidad a 11\% y el sedentarismo a $76 \%{ }^{9}$. También es preocupante la salud mental de los jóvenes, ya que $18,1 \%$ de los adolescentes declara haber tenido algún intento suicida o de auto-agresión durante el último año ${ }^{8}$.

El abordaje de estas problemáticas en Chile no es fácil. Si bien ha habido avances en la atención 
y cobertura en adolescentes mayores de 15 años, el grupo etario de 10 a 14 años ha quedado más desprovisto ${ }^{10}$. Sólo recientemente se ha presentado el Programa Nacional de Salud Integral de Adolescentes y Jóvenes en Chile, que contempla entre sus metas reducir factores de riesgo para las ECNTs y lograr una cobertura a todo el rango etario de adolescentes para el $2020^{10}$.

Pareciera ser que en Chile el sistema escolar es un contexto propicio para implementar intervenciones costo-efectivas para la promoción de conductas saludables, dado el alto nivel de escolarización alcanzado. Por otro lado, existe evidencia que la exposición a un clima escolar positivo disminuye el uso de sustancias de abuso $y$ embarazo adolescente ${ }^{11}$ e intervenciones que mejoran el vínculo entre los alumnos y la escuela han sido efectivas en la disminución de la violencia y mejoría en la actividad física ${ }^{12}$.

En Chile no existen estudios que hayan evaluado la conductas promotoras de salud en adolescentes tempranos (10-14 años), ni tampoco los factores que pudieran estar relacionados con estas prácticas.

A nivel internacional existen instrumentos que permiten evaluar las conductas promotoras de salud, como: Health-Promoting Lifestyle (HPLP) ${ }^{13}$, Adolescent Lifestyle Questionnaire (ALQ) ${ }^{14}$, Adolescent Lifestyle Profile (ALP) ${ }^{15}$ y el Adolescent Health-Promoting (AHP) scale ${ }^{16}$. La AHP es una de las más usadas, ha sido traducida a diferentes idiomas ${ }^{17}$ y utilizada para evaluar los efectos de la educación en salud ${ }^{17-19}$.

El objetivo de este estudio fue medir la prevalencia de conductas promotoras de salud y determinar los factores que se asocian a éstas, en una población de adolescentes que se encuentran cursando entre los niveles quinto a octavo de escolaridad básica.

\section{Sujetos y Método}

\section{Participantes}

Se solicitó a la Dirección de Administración de Educación Municipal de San Felipe el listado de colegios de la ciudad. Para la selección de la muestra se estratificó la población de alumnos de $5^{\circ}$ a $8^{\circ}$ Básicos de colegios de San Felipe de acuerdo a tres variables: Sexo (Mujeres y Hombres); nivel socioeconómico (NSE), de acuerdo a los criterios del Sistema de Medición de los Resultados de Aprendizaje (Alto, Medio y Bajo); y nivel de curso ( $5^{\circ}$ a $8^{\circ}$ Básicos). Se proyectó una participación de 60 alumnos en cada una de las celdas de la estratificación para lograr la mayor representatividad; sin embargo, dos colegios de NSE Alto no aceptaron participar. Participaron 1.465 estudiantes. La no participación estuvo dada por la falta de consentimiento de los padres y ausentismo el día de aplicación de la encuesta.

\section{Instrumentos}

1. Escala de Promoción de Salud del Adolescente $(A H P)^{16}$ : Mide conductas promotoras de salud tales como responsabilidad en salud y nutrición; ejercicio físico y manejo del estrés; apreciación de la vida y apoyo social. Esta escala fue desarrollada por Chen et al., y se basa en el Modelo Promotor de Salud (MPS) de Nola Pender ${ }^{3}$. Tiene 40 afirmaciones, con respuestas que van de $1=$ nunca a $5=$ siempre. Se usó el puntaje total obtenido de la sumatoria de los ítems. Para su interpretación, y siguiendo la recomendación de Chen et al. ${ }^{17}$, se recodificó como variable dicotómica para cada alumno según si su puntaje estaba por sobre o por debajo el promedio de la muestra total (140 puntos). Los autores cuentan con la autorización para su adaptación y validación en Chile.

2. Escala de Conductas de Riesgo en Adolescentes (ECRA): Evalúa características sociodemográficas, familiares y del sistema escolar de los adolescentes; percepción de los jóvenes de la propia salud, percepción de conductas de riesgo en grupo de pares; uso de sustancias químicas por el propio adolescente, por sus pares y padres; entre otras. Validada en Chile ${ }^{20,21}$. Tiene 70 preguntas. (Tabla 1).

3. Cuestionario del Programa de Habilidades para la Vida (LSTQ-MS)22: Evalúa el conocimiento, actitudes y habilidades de resistencia al consumo de sustancias de abuso. Para este estudio se utilizaron 2 sub-escalas, "Opinión sobre el consumo de alcohol y cigarrillos" y "Reacción ante la presión de consumo" (Tabla 1).

4. Escala de membrecía escolar ${ }^{23}$ : Mide nivel de pertenencia que el alumno refiere en relación a su colegio. La adaptación usada en Chile tiene 13 ítems con 5 niveles de respuesta $(1=$ No es verdad en absoluto, a 5 = Completamente verdad). Los autores cuentan con la autorización de su uso en Chile (Tabla 1). 
Factores asociados a conductas promotoras de salud en adolescentes chilenos - J. Gaete et al

Tabla 1. Variables independientes, según dominio personal, familiar y escolar

\begin{tabular}{|c|c|c|c|c|}
\hline $\begin{array}{l}\text { Nombre del } \\
\text { constructo }\end{array}$ & $\begin{array}{l}\text { n de } \\
\text { ítems }\end{array}$ & Respuesta/Rango & $\begin{array}{l}\text { Alfa de } \\
\text { Cronbach }\end{array}$ & $\begin{array}{c}\text { Cuestionario } \\
\text { de donde } \\
\text { procede }\end{array}$ \\
\hline \multicolumn{5}{|l|}{ Personal } \\
\hline Sexo & 1 & $1=$ Hombre; 2 = Mujer & & ECRA \\
\hline Curso & 1 & $\begin{array}{l}1=5^{\circ} \text { Básico } \\
2=6^{\circ} \text { Básico } \\
3=7^{\circ} \text { Básico } \\
4=8^{\circ} \text { Básico }\end{array}$ & & ECRA \\
\hline Rendimiento & 1 & $\begin{array}{l}1=<4,0 \\
2=4,0-4,4 \\
3=4,5-4,9 \\
4=5,0-5,4 \\
5=5,5-5,9 \\
6=6,0-6,4 \\
7=6,5-7,0\end{array}$ & & ECRA \\
\hline Religiosidad & 1 & $\begin{array}{l}1=\text { Nada religioso } \\
2=\text { Poco religioso } \\
3=\text { Muy religioso }\end{array}$ & & ECRA \\
\hline $\begin{array}{l}\text { Conformidad con } \\
\text { peso }\end{array}$ & 1 & $\begin{array}{l}1 \text { = Nada conforme } \\
2=\text { Más o menos conforme } \\
3 \text { = Muy conforme }\end{array}$ & & ECRA \\
\hline $\begin{array}{l}\text { Conformidad con } \\
\text { apariencia física }\end{array}$ & 1 & $\begin{array}{l}1 \text { = Nada conforme } \\
2=\text { Más o menos conforme } \\
3 \text { = Muy conforme }\end{array}$ & & ECRA \\
\hline Percepción de salud & 1 & $\begin{array}{l}1=\text { No tan sano como otros } \\
2=\text { Igual de sano que otros } \\
3=\text { Más sano que otros }\end{array}$ & & ECRA \\
\hline $\begin{array}{l}\text { Escala de } \\
\text { Problemas de } \\
\text { conducta }\end{array}$ & 6 & $\begin{array}{l}\text { Ejemplo de ítem: } \\
\text { "Durante el último año, cuántas veces copiaste en las pruebas" } \\
\text { Respuesta: } \\
1 \text { = Nunca } \\
2 \text { = De uno a dos veces } \\
3 \text { = De tres a } 5 \text { veces } \\
4=\text { De } 6 \text { a } 10 \text { veces } \\
5 \text { = Más de } 10 \text { veces } \\
\text { Rango de puntaje de escala: } 6 \text { a } 30 \text { puntos }\end{array}$ & 0,65 & ECRA \\
\hline $\begin{array}{l}\text { Control de } \\
\text { conducta }\end{array}$ & 1 & $\begin{array}{l}1=\text { No, y me preocupa mucho } \\
2=\text { No, y me preocupa poco } \\
3=\text { Sí, sólo alguna veces } \\
4=\text { Sí, todo el tiempo }\end{array}$ & & ECRA \\
\hline $\begin{array}{l}\text { Estabilidad } \\
\text { emocional }\end{array}$ & 1 & $\begin{array}{l}1=\text { Nunca } \\
2=\text { Algunas veces } \\
3=\text { Todo el tiempo }\end{array}$ & & ECRA \\
\hline Desánimo & 1 & $\begin{array}{l}1=\text { Extremadamente } \\
2=\text { Bastante } \\
3=\text { Un poco } \\
4=\text { Nada }\end{array}$ & & ECRA \\
\hline Ansiedad & 1 & $\begin{array}{l}1=\text { Todo el tiempo } \\
2=\text { Algunas veces } \\
3=\text { Nunca }\end{array}$ & & ECRA \\
\hline
\end{tabular}


Factores asociados a conductas promotoras de salud en adolescentes chilenos - J. Gaete et al

\begin{tabular}{|c|c|c|c|c|}
\hline $\begin{array}{l}\text { Escala de Opinión } \\
\text { sobre el consumo } \\
\text { de alcohol y } \\
\text { cigarrillos }\end{array}$ & 6 & $\begin{array}{l}\text { Ejemplo de ítem: } \\
\text { "El fumar cigarrillos hace que te veas bacán" } \\
1=\text { Muy en desacuerdo } \\
2=\text { En desacuerdo } \\
3 \text { = Ni uno ni lo otro } \\
4=\text { De acuerdo } \\
5=\text { Muy de acuerdo } \\
\text { Rango de puntaje de escala: } 6 \text { a } 30 \text { puntos }\end{array}$ & 0,88 & LSTQ \\
\hline $\begin{array}{l}\text { Escala de Reacción } \\
\text { ante la presión de } \\
\text { consumo }\end{array}$ & 5 & $\begin{array}{l}\text { Ejemplo de ítem: } \\
\text { "Qué dirías cuando alguien te presiona a que fumes cigarrillos" } \\
\text { Respuestas: } \\
1 \text { = Definitivamente diría que no } \\
2 \text { = Probablemente diría que no } \\
3 \text { = No estoy seguro } \\
4 \text { = Probablemente diría que sí } \\
5 \text { = Definitivamente diría que sí } \\
\text { Rango de puntaje de escala: } 5 \text { a } 25 \text { puntos }\end{array}$ & 0,90 & LSTQ \\
\hline \multicolumn{5}{|l|}{ Familiar } \\
\hline $\begin{array}{l}\text { Padres viven juntos } \\
\text { o no }\end{array}$ & 1 & $\begin{array}{l}0=\text { No vive con ambos padres } \\
1=\text { Vive con ambos padres }\end{array}$ & & ECRA \\
\hline $\begin{array}{l}\text { Educación del } \\
\text { padre }\end{array}$ & 1 & $\begin{array}{l}0=12 \text { o menos años } \\
1=13 \text { o más años }\end{array}$ & & ECRA \\
\hline $\begin{array}{l}\text { Educación de la } \\
\text { madre }\end{array}$ & 1 & $\begin{array}{l}0=12 \text { o menos años } \\
1=13 \text { o más años }\end{array}$ & & ECRA \\
\hline Trabajo del padre & 1 & $\begin{array}{l}1=\text { No trabaja } \\
2=\text { Trabaja tiempo parcial } \\
3=\text { Trabaja tiempo completo }\end{array}$ & & ECRA \\
\hline Trabajo de la madre & 1 & $\begin{array}{l}1=\text { No trabaja } \\
2=\text { Trabaja tiempo parcial } \\
3=\text { Trabaja tiempo completo }\end{array}$ & & ECRA \\
\hline $\begin{array}{l}\text { Historia de uso de } \\
\text { drogas en padres }\end{array}$ & 1 & $\begin{array}{l}1=\text { Sí } \\
2=\text { No sé } \\
3=\text { No }\end{array}$ & & ECRA \\
\hline $\begin{array}{l}\text { Psicopatología } \\
\text { Parental }\end{array}$ & 1 & $\begin{array}{l}1=\text { Sí } \\
2=\text { No sé } \\
3=\text { No }\end{array}$ & & ECRA \\
\hline $\begin{array}{l}\text { Alcoholismo } \\
\text { parental }\end{array}$ & 1 & $\begin{array}{l}1=\text { Sí } \\
2=\text { No sé } \\
3=\text { No }\end{array}$ & & ECRA \\
\hline \multicolumn{5}{|l|}{ Escolar } \\
\hline $\begin{array}{l}\text { Escala de Ambiente } \\
\text { escolar negativo }\end{array}$ & 3 & $\begin{array}{l}\text { Ejemplo de ítem: } \\
\text { "En tu opinión, ¿has visto o te han contado que estudiantes } \\
\text { roben en tu colegio? } \\
\text { Respuesta: } \\
1=\text { Ocurre mucho } \\
2=\text { Ocurre poco } \\
3=\text { No ocurre }\end{array}$ & 0,74 & ECRA \\
\hline
\end{tabular}


Tabla 1. Variables independientes, según dominio personal, familiar y escolar (continuación)

\begin{tabular}{|c|c|c|c|c|}
\hline $\begin{array}{l}\text { Ambiente escolar } \\
\text { asociado al uso de } \\
\text { substancias }\end{array}$ & 1 & $\begin{array}{l}1=\text { Ocurre mucho } \\
2=\text { Ocurre poco } \\
3=\text { No ocurre }\end{array}$ & & ECRA \\
\hline $\begin{array}{l}\text { Ambiente escolar } \\
\text { asociado a uso de } \\
\text { alcohol }\end{array}$ & 1 & $\begin{array}{l}1=\text { Ocurre mucho } \\
2=\text { Ocurre poco } \\
3=\text { No ocurre }\end{array}$ & & ECRA \\
\hline $\begin{array}{l}\text { Escala de } \\
\text { Membrecía } \\
\text { escolar }\end{array}$ & 13 & $\begin{array}{l}\text { Ejemplo de ítem: } \\
\text { "Me siento parte de este colegio" } \\
\text { Respuestas: } \\
1 \text { = No es verdad en absoluto } \\
2 \text { = Algo verdadera } \\
3=\text { A veces verdadera } \\
4=\text { Casi siempre verdadera } \\
5=\text { Completamente verdadera } \\
\text { Rango de puntaje de escala: } 13 \text { a } 65 \text { puntos }\end{array}$ & 0,89 & PSSM \\
\hline $\begin{array}{l}\text { Escala de } \\
\text { Compromiso } \\
\text { académico }\end{array}$ & 5 & $\begin{array}{l}\text { Ejemplo de ítem: } \\
\text { "Me gustan los ramos (o asignaturas) de este año" } \\
\text { Respuesta: } \\
1 \text { = iNO! } \\
2 \text { = no } \\
3 \text { = sí } \\
\begin{array}{l}4=\text { iSí! } \\
\text { Rango de puntaje de escala: } 5 \text { a } 20 \text { puntos }\end{array}\end{array}$ & 0,78 & SB \\
\hline
\end{tabular}

5. Escala de compromiso académico ${ }^{24}$ : Mide nivel de vinculación del alumno con el colegio y, especialmente, el compromiso que el alumno tiene con sus actividades académicas. Tiene 5 ítems, con 4 niveles de respuesta $\left(1={ }_{i}\right.$ NO! a $4={ }_{¡}$ SÍ! $)($ Tabla 1$)$.

\section{Análisis estadístico}

Se utilizaron estadísticos descriptivos generales para mostrar la distribución de distintas variables en la población en estudio.

Las asociaciones fueron estudiadas a través de modelos de regresión logística.

Las variables independientes surgieron de los cuestionarios ECRA y LSTQ-MS. Los ítems fueron considerados en forma independiente, y cuando un constructo de interés fue evaluado por varios ítems se generaron escalas. Como variables independientes se consideraron las escalas de membrecía escolar y compromiso académico. Todas estas variables se ordenaron en 3 contextos de influencia: individual, familiar y escolar.

En relación a la edad, se utilizó como variable proxy el nivel del curso del alumno, ya que es es un indicador confiable de la edad y fue obtenida para todos los casos a través de los colegios.

La variable dependiente correspondió a la escala de Promoción de Salud de Adolescentes. (Tabla 4). En el modelo 0 se establecieron los OR (95\% IC) en forma univariada. En el modelo 1 se consideraron las variables por contexto de influencia en forma separada, para determinar secuencialmente el efecto de las variables del mismo contexto ajustadas entre ellas. Los factores que presentaron una asociación con valores $\mathrm{p} \leq 0,2$ fueron considerados para el modelo final. Este último incluyó todos los factores asociados en los tres contextos de influencia, y se consideró un nivel de significación correspondiente al $\mathrm{p} \leq 0,05$.

Todos los análisis se realizaron en STATA 12.01 .

\section{Consideraciones éticas}

El estudio fue aprobado por el comité de ética de la Facultad de Educación de la Pontificia Universidad Católica de Chile. Los participantes requirieron el consentimiento escrito de parte de sus padres y el asentimiento de ellos. Toda la información fue manejada con estricta confidencialidad 
Factores asociados a conductas promotoras de salud en adolescentes chilenos - J. Gaete et al

y los colegios participantes fueron informados de los resultados a nivel colectivo.

\section{Resultados}

\section{Descriptivos generales}

La muestra correspondió a 1.465 alumnos, distribuidos equitativamente en los cuatro niveles escolares estudiados en siete colegios de la ciudad de San Felipe. Es necesario mencionar que la no participación de dos colegios de NSE Alto hizo que el número de alumnos elegidos para este seg- mento fuera menor (Alto, Matrícula $=391$; Medio, Matrícula $=834 ;$ Bajo, Matrícula $=883$ ). Por otro lado, al momento de calcular la participación de los alumnos, los del NSE Alto fueron los que más consintieron: 82,4\%; Medio: 53,5\%; y Bajo, $78,9 \%$. Dentro de los participantes $45,1 \%$ fueron mujeres y $64,8 \%$ declaró vivir con ambos padres. La mayoría de los alumnos dijeron ser católicos $(77,4 \%)$. Cerca de $27 \%$ de los alumnos refirió que su padre y madre han cursado estudios después de la enseñanza media. El 74\% de los alumnos refirió que su padre trabaja tiempo completo, y 56,5\% lo dijo de su madre (Tabla 2).

Tabla 2. Descriptivos generales del total de la muestra y por niveles socio-económicos (Bajo, Medio y Alto)

\begin{tabular}{|c|c|c|c|c|c|c|}
\hline \multirow{2}{*}{$\begin{array}{l}\text { Variable } \\
\text { Mujeres }\end{array}$} & & \multicolumn{2}{|c|}{$\begin{array}{l}\text { Total } \\
\%(n)\end{array}$} & \multirow{2}{*}{$\begin{array}{c}\text { Bajo } \\
\% \text { (n) } \\
40,7 \quad(275)\end{array}$} & \multirow{2}{*}{$\begin{array}{l}\text { Medio } \\
\% \text { (n) } \\
48,9(229)\end{array}$} & \multirow{2}{*}{$\begin{array}{c}\text { Alto } \\
\% \text { (n) } \\
50,0(156)\end{array}$} \\
\hline & & 45,1 & $(660)$ & & & \\
\hline Curso & $\begin{array}{l}\text { Quinto básico } \\
\text { Sexto básico } \\
\text { Séptimo básico } \\
\text { Octavo básico }\end{array}$ & $\begin{array}{l}27,5 \\
25,6 \\
25,9 \\
21,0\end{array}$ & $\begin{array}{l}(403) \\
(375) \\
(379) \\
(308)\end{array}$ & $\begin{array}{ll}22,5 & (154) \\
24,1 & (165) \\
29,2 & (200) \\
24,1 & (165)\end{array}$ & $\begin{array}{ll}34,4 & (161) \\
29,7 & (139) \\
20,5 & (98) \\
15,0 & (70)\end{array}$ & $\begin{array}{cc}28,1 & (88) \\
22,7 & (71) \\
25,9 & (81) \\
23,3 & (73)\end{array}$ \\
\hline $\begin{array}{l}\text { Conductas promotoras } \\
\text { de salud }\end{array}$ & $\begin{array}{l}\text { Bajo }(B)=\text { Puntaje } \leq 140 \\
\text { Sobre }(S)=\text { Puntaje }>140\end{array}$ & $\begin{array}{l}\text { B: } 45, \\
\text { S: } 54,\end{array}$ & $\begin{array}{l}1(661) \\
9(804)\end{array}$ & $\begin{array}{l}\text { B: } 47,4(324) \\
\text { S: } 52,6(360)\end{array}$ & $\begin{array}{l}\text { B: } 48,3(226) \\
\text { S: } 51,7(242)\end{array}$ & $\begin{array}{l}\text { B: } 35,5(111) \\
\text { S: } 64,5(202)\end{array}$ \\
\hline $\begin{array}{l}\text { Condición de } \\
\text { los padres }\end{array}$ & $\begin{array}{l}\text { Casados } \\
\text { Separados } \\
\text { Divorciados } \\
\text { Conviven } \\
\text { Padres no se casaron ni conviven } \\
\text { Uno o ambos fallecieron } \\
\text { No sabe }\end{array}$ & $\begin{array}{r}44,9 \\
16,9 \\
4,7 \\
17,6 \\
10,6 \\
1,8 \\
3,6\end{array}$ & $\begin{array}{r}(654) \\
(246) \\
(68) \\
(257) \\
(154) \\
(26) \\
(53)\end{array}$ & $\begin{array}{rr}38,6 & (262) \\
18,4 & (125) \\
4,0 & (27) \\
19,2 & (130) \\
13,8 & (94) \\
2,0 & (14) \\
4,0 & (27)\end{array}$ & $\begin{array}{rr}41,6 & (194) \\
19,3 & (90) \\
3,9 & (18) \\
20,6 & (96) \\
8,4 & (39) \\
2,2 & (10) \\
4,0 & (19)\end{array}$ & $\begin{array}{rr}63,3 & (198) \\
10,0 & (31) \\
7,4 & (23) \\
9,9 & (31) \\
6,7 & (21) \\
0,6 & (2) \\
2,2 & (7)\end{array}$ \\
\hline Vive con ambos padres & Sí & 64,8 & $(911)$ & $60,1(392)$ & $64,9(290)$ & $74,8(229)$ \\
\hline Religión & $\begin{array}{l}\text { Católica } \\
\text { Evangélica } \\
\text { Mormona } \\
\text { Testigos de Jehová } \\
\text { Judía } \\
\text { Otra } \\
\text { Ninguna }\end{array}$ & $\begin{array}{r}77,4 \\
11,8 \\
1,3 \\
1,7 \\
0,1 \\
1,6 \\
6,1\end{array}$ & $\begin{array}{r}1.119) \\
(170) \\
(19) \\
(25) \\
(1) \\
(23) \\
(88)\end{array}$ & $\begin{array}{rr}70,6 & (477) \\
15,0 & (102) \\
1,9 & (13) \\
2,4 & (16) \\
0,2 & (1) \\
1,8 & (12) \\
8,1 & (55)\end{array}$ & $\begin{array}{rr}77,5 & (355) \\
12,9 & (59) \\
1,3 & (6) \\
1,7 & (8) \\
0,0 & (0) \\
1,3 & (6) \\
5,2 & (24)\end{array}$ & $\begin{array}{rr}92,2 & (287) \\
2,9 & (9) \\
0,0 & (0) \\
0,3 & (1) \\
0,0 & (0) \\
1,6 & (5) \\
2,9 & (9)\end{array}$ \\
\hline Educación padre & $\begin{array}{l}12 \text { años o menos } \\
13 \text { años o más }\end{array}$ & $\begin{array}{l}72,6 \\
27,4\end{array}$ & $\begin{array}{l}(777) \\
(293)\end{array}$ & $\begin{array}{l}85,4(433) \\
14,6 \quad(74)\end{array}$ & $\begin{array}{l}85,4(272) \\
17,6 \quad(58)\end{array}$ & $\begin{array}{r}30,9 \quad(72) \\
69,1(161)\end{array}$ \\
\hline Educación madre & $\begin{array}{l}12 \text { años o menos } \\
13 \text { años o más }\end{array}$ & $\begin{array}{l}72,9 \\
27,1\end{array}$ & $\begin{array}{l}(851) \\
(316)\end{array}$ & $\begin{array}{l}86,8(473) \\
13,2 \quad(72)\end{array}$ & $\begin{array}{l}81,8(297) \\
18,2 \quad(66)\end{array}$ & $\begin{array}{l}31,3 \quad(81) \\
68,7(178)\end{array}$ \\
\hline Ocupación padre & $\begin{array}{l}\text { No trabaja } \\
\text { Trabaja medio tiempo } \\
\text { Trabaja tiempo completo }\end{array}$ & $\begin{array}{r}4,4 \\
21,6 \\
74,0\end{array}$ & $\begin{array}{r}(58) \\
(288) \\
(987)\end{array}$ & $\begin{array}{r}5,0 \quad(31) \\
21,0(128) \\
73,9(451)\end{array}$ & $\begin{array}{rr}4,6 & (20) \\
22,8 & (98) \\
72,3 & (312)\end{array}$ & $\begin{array}{rr}2,4 & (7) \\
21,2 & (62) \\
76,5 & (224)\end{array}$ \\
\hline Ocupación madre & $\begin{array}{l}\text { No trabaja } \\
\text { Trabaja medio tiempo } \\
\text { Trabaja tiempo completo }\end{array}$ & $\begin{array}{l}12,9 \\
30,7 \\
56,5\end{array}$ & $\begin{array}{l}(140) \\
(334) \\
(615)\end{array}$ & $\begin{array}{r}14,7(72) \\
31,4(154) \\
54,0(264)\end{array}$ & $\begin{array}{r}15,0(52) \\
31,4(109) \\
53,6(186)\end{array}$ & $\begin{array}{rr}6,4 & (16) \\
28,1 & (71) \\
23,1 & (252)\end{array}$ \\
\hline
\end{tabular}


Según NSE (Tabla 2), se observan ciertas diferencias en la muestra. En el NSE bajo encontramos que $60,1 \%$ de alumnos viven con ambos padres, que $14,6 \%$ de los padres y $13,2 \%$ de las madres alcanzaron estudios más allá de la enseñanza media. Además, en esta categoría encontramos el mayor porcentaje de madres que trabaja a tiempo completo $(54,0 \%)$. En relación a las conductas promotoras de salud, $64,5 \%$ de los alumnos del NSE alto, estaban por sobre el promedio, estimador que fue sólo de 52,6\% en el NSE bajo.

\section{Factores asociados a conductas promotoras de salud}

En un análisis inicial univariado, a mayor nivel de curso disminuyó el porcentaje de alumnos con conductas promotoras de salud por sobre el promedio (Tabla 3 ) (5º́sico: $62,3 \%$ versus $8^{\circ}$ Básico: $46,7 \%, p=0,001)$. Además, las mujeres y los alumnos que perciben tener mejor estado de salud, tuvieron niveles más altos de conductas promotoras de salud (Tabla 3 ).

En los análisis de regresión logística, en el modelo univariado (modelo 0 ), se observan varios factores que se asocian a tener conductas promotoras de salud por sobre el promedio de la muestra (Tabla 4). En el estudio multivariado, modelo final, a nivel personal resultaron positivamente asociadas las variables de rendimiento académico $(\mathrm{OR}=1,18$; 95\% IC 1,01-1,38), percepción del estado de salud $(\mathrm{OR}=1,44 ; 95 \%$ IC: $1,07-1,95)$ y conformidad con la apariencia física (OR $=1,39 ; 95 \%$ CI: 1,00-1,92). En el contexto familiar no hubo variables asociadas. Finalmente, las variables membrecía escolar (OR $=1,05$; 95\% IC: $1,03-1,07)$ y compromiso académico $(\mathrm{OR}=1,27 ; 95 \%$ IC: $1,18-1,35)$, resultaron positivamente asociadas a conductas promotoras de salud en el contexto escolar (Tabla 4).

Tabla 3. Distribución de conductas promotoras de salud, por sobre (Alto) y por debajo (Bajo) del promedio de la muestra

\begin{tabular}{|c|c|c|c|c|}
\hline Factores asociados & & Bajo & Alto & valor $p\left(\chi^{2}\right)$ \\
\hline Sexo & $\begin{array}{l}\text { Mujer } \\
\text { Hombre }\end{array}$ & $\begin{array}{l}40,45 \\
49,07\end{array}$ & $\begin{array}{l}59,55 \\
50,93\end{array}$ & 0,001 \\
\hline Curso & $\begin{array}{l}5^{\circ} \\
6^{\circ} \\
7^{\circ} \\
8^{\circ}\end{array}$ & $\begin{array}{l}37,72 \\
45,07 \\
46,44 \\
53,25\end{array}$ & $\begin{array}{l}62,28 \\
54,93 \\
53,56 \\
46,72\end{array}$ & 0,001 \\
\hline NSE & $\begin{array}{l}\text { Alto } \\
\text { Medio } \\
\text { Bajo }\end{array}$ & $\begin{array}{l}35,46 \\
48,29 \\
47,37\end{array}$ & $\begin{array}{l}64,65 \\
51,71 \\
52,63\end{array}$ & 0,001 \\
\hline Promedio de notas año anterior & $\begin{array}{l}6,5-7,0 \\
6,0-6,4 \\
5,5-5,9 \\
5,0-5,4 \\
4,5-4,9 \\
4,0-4,4 \\
\text { Menos de 4,0 }\end{array}$ & $\begin{array}{l}24,66 \\
38,50 \\
47,16 \\
59,26 \\
56,14 \\
70,00 \\
70,59\end{array}$ & $\begin{array}{l}75,34 \\
61,50 \\
52,84 \\
40,74 \\
43,86 \\
30,00 \\
29,41\end{array}$ & 0,000 \\
\hline Percepción del estado de salud & $\begin{array}{l}\text { Mejor } \\
\text { Promedio } \\
\text { Peor }\end{array}$ & $\begin{array}{l}36,90 \\
44,67 \\
44,94\end{array}$ & $\begin{array}{l}63,10 \\
55,33 \\
55,06\end{array}$ & 0,000 \\
\hline Educación padre & $\begin{array}{l}12 \text { años o menos } \\
13 \text { años o más }\end{array}$ & $\begin{array}{l}46,07 \\
36,86\end{array}$ & $\begin{array}{l}53,93 \\
63,14\end{array}$ & 0,007 \\
\hline Educación madre & $\begin{array}{l}12 \text { años o menos } \\
13 \text { años o más }\end{array}$ & $\begin{array}{l}44,77 \\
38,29\end{array}$ & $\begin{array}{l}55,23 \\
61,71\end{array}$ & 0,047 \\
\hline Con quien vive & $\begin{array}{l}\text { Con ambos padres } \\
\text { Sin ambos padres }\end{array}$ & $\begin{array}{l}40,94 \\
49,73\end{array}$ & $\begin{array}{l}59,06 \\
50,21\end{array}$ & 0,002 \\
\hline
\end{tabular}


Factores asociados a conductas promotoras de salud en adolescentes chilenos - J. Gaete et al

Tabla 4. Resultados de la asociación de factores personales, familiares y escolares y el tener conductas promotoras de salud por sobre el promedio usando modelos de regresión logística

\begin{tabular}{|c|c|c|c|c|c|c|}
\hline \multirow[b]{2}{*}{ Variable predictora } & \multicolumn{2}{|c|}{ Modelo 0* } & \multicolumn{2}{|c|}{ Modelo $1^{\dagger}$} & \multicolumn{2}{|c|}{ Modelo Finalł } \\
\hline & OR & $95 \%$ IC & OR & $95 \%$ IC & OR & $95 \%$ IC \\
\hline \multicolumn{7}{|l|}{ Variables personales } \\
\hline Nivel socio económico & 1,22 & $1,07-1,40$ & 1,02 & $0,87-1,97$ & & \\
\hline Sexo & 1,41 & $1,15-1,74$ & 1,38 & $1,08-1,78$ & 1,38 & $0,95-2,00$ \\
\hline Religiosidad & 1,57 & $1,30-1,91$ & 1,30 & $1,04-1,63$ & 1,29 & $0,91-1,83$ \\
\hline Curso & 0,82 & $0,75-0,90$ & 0,95 & $0,85-1,06$ & & \\
\hline Rendimiento & 1,43 & $1,32-1,56$ & 1,30 & $1,17-1,43$ & $1,18^{* *}$ & $1,01-1,38$ \\
\hline Conformidad con peso & 1,42 & $1,23-1,63$ & 1,02 & $0,85-1,23$ & & \\
\hline Conformidad con apariencia física & 1,77 & $1,48-2,13$ & 1,42 & $1,12-1,80$ & $1,39 * *$ & $1,00-1,92$ \\
\hline Estado salud & 1,67 & $1,41-1,97$ & 1,47 & $1,21-1,79$ & $1,44^{* *}$ & $1,07-1,95$ \\
\hline Escala de problemas de conducta & 0,87 & $0,84-0,90$ & 0,94 & $0,90-0,98$ & 0,99 & $0,93-1,06$ \\
\hline Control de conducta & 1,58 & $1,37-1,81$ & 1,31 & $1,12-1,54$ & 1,06 & $0,82-1,38$ \\
\hline Estabilidad emocional & 1,82 & $1,53-2,16$ & 1,44 & $1,17-1,76$ & 1,25 & $0,90-1,73$ \\
\hline Ánimo & 1,42 & $1,25-1,62$ & 1,05 & $0,88-1,24$ & & \\
\hline Ansiedad & 1,21 & $0,99-1,48$ & 1,17 & $0,91-1,50$ & & \\
\hline $\begin{array}{l}\text { Escala de opinión sobre el consumo de alcohol y } \\
\text { cigarrillo }\end{array}$ & 0,95 & $0,93-0,97$ & 0,97 & $0,95-1,00$ & 0,97 & $0,93-1,01$ \\
\hline Escala de reacción frente a presiones de consumo & 0,93 & $0,89-0,97$ & 0,96 & $0,92-1,01$ & 0,99 & $0,92-1,07$ \\
\hline \multicolumn{7}{|l|}{ Variables familiares } \\
\hline Padres viven juntos o no & 1,50 & $1,20-1,87$ & 1,13 & $0,80-1,61$ & & \\
\hline Educación del padre & 1,46 & $1,11-1,92$ & 2,26 & $1,41-3,60$ & 1,62 & $0,97-2,71$ \\
\hline Trabajo del padre & 1,13 & $0,93-1,38$ & 1,33 & $0,99-1,80$ & 1,19 & $0,84-1,69$ \\
\hline Educación de la madre & 1,30 & $1,00-1,70$ & 0,77 & $0,48-1,21$ & 0,82 & $0,49-1,38$ \\
\hline Trabajo de la madre & 1,03 & $0,87-1,22$ & 0,88 & $0,69-1,11$ & & \\
\hline Historia de uso de drogas en padres & 1,58 & $1,31-1,91$ & 1,00 & $0,66-1,50$ & & \\
\hline Psicopatología parental & 1,47 & $1,23-1,77$ & 0,72 & $0,52-0,99$ & 0,81 & $0,52-1,26$ \\
\hline $\begin{array}{l}\text { Alcoholismo parental } \\
\text { Variables escolares }\end{array}$ & 1,88 & $1,45-2,30$ & 0,83 & $0,57-1,23$ & 0,87 & $0,51-1,50$ \\
\hline Ambiente escolar negativo & 1,08 & $1,01-1,16$ & 1,09 & $1,00-1,20$ & 0,98 & $0,87-1,11$ \\
\hline Ambiente escolar asociado a uso de sustancias & 1,58 & $1,16-2,14$ & 0,84 & $0,55-1,29$ & & \\
\hline Ambiente asociado a uso de alcohol & 1,21 & $0,97-1,51$ & 0,87 & $0,65-1,18$ & & \\
\hline Escala de membrecía escolar & 1,08 & $1,07-1,09$ & 1,06 & $1,04-1,07$ & $1,05^{* *}$ & $1,03-1,07$ \\
\hline Escala de compromiso académico & 1,33 & $1,28-1,39$ & 1,23 & $1,18-1,29$ & $1,27^{* *}$ & $1,18-1,35$ \\
\hline
\end{tabular}

Nota: *Modelo 0: Regresión logística univariada o no ajustada. ${ }^{\dagger}$ Modelo 1: Regresión logística multivariada por dominio. Todas las variables de cada dominio (personal, familiar y escolar) se incluyeron en la regresión logística. ‘’Modelo final: Regresión logística multivariada. Este modelo incluyó todas las variables que tuvieron un valor $p<0,2$ de asociación en el Modelo 1. Se muestra con $\left(^{* *}\right)$ las variables que resultaron con una asociación con un valor $p<0,05$.

\section{Discusión}

Según nuestra información, éste es el primer estudio en Chile que evalúa la frecuencia de conductas promotoras de salud en la población escolar adolescente entre $5^{\circ}$ y $8^{\circ}$ Básicos y sus factores asociados. Encontramos que los alumnos con mayor frecuencia de conductas promotoras de salud fueron aquellos con un mejor rendimiento y compromiso académico, mejor percepción de su estado de salud y conformidad con su apariencia física y mayor sentido de pertenencia al colegio.

Inicialmente parecía haber una relación con el nivel del curso, el sexo y otros factores medidos (modelo 0); sin embargo, esto cambia cuando las distintas variables se van ajustando con otras del mismo contexto (modelo 1) o de otros contextos (modelo final). Muchas de las asociaciones encontradas inicialmente probablemente se debían al efecto confundente de otras variables. 
Sin embargo, a nivel personal, parece claro que los estudiantes que presentaron mayores frecuencias de conductas promotoras de salud fueron aquellos que tuvieron: (i) una mejor percepción de su estado de salud; (ii) una mayor conformidad con su apariencia física; y (iii) mejores notas. El Modelo Promotor de Salud de Pender ${ }^{3}$ plantea que uno de los determinantes individuales del desarrollo de conductas promotoras de salud es el estado de salud percibido, lo que es congruente con nuestros resultados ${ }^{25}$. Otro estudio realizado en Chile en población general de 15 a 65 años, también encontró esta misma asociación ${ }^{26}$. Sin embargo, García et al, estudiando el efecto de esta variable en el desarrollo de actividad física, encontró resultados negativos en una población similar a la de nuestro estudio ${ }^{27}$ y diferencias entre géneros sobre el desarrollo de estas conductas; algo que nosotros no encontramos. Esto se podría explicar por diferencias culturales, lo que da aun mayor relevancia a nuestro estudio, estableciendo la necesidad de evaluar estas variables y su desarrollo en población chilena. Por otro lado, la variable "conformidad con apariencia física" podría ser considerada como una variable proxy de autoestima, factor importante al momento de considerar los determinantes del desarrollo de conductas promotoras de salud según Pender, y realzando la importancia del efecto de los niveles de autestima y autoeficacia planteado por algunos autores $^{25,26}$. Finalmente, en relación al rendimiento académico, nos parece plausible que alumnos con mejores notas tengan, a su vez, una mayor capacidad de auto-regulación que les ayudaría a desarrollar conductas promotoras de salud, las que requieren esfuerzo ${ }^{28}$.

A nivel familiar, no encontramos variables asociadas. Es llamativo que variables como la educación de los padres no hayan tenido una influencia.

A nivel escolar, se encontraron claramente asociados a la presencia de conductas promotoras de salud: i) membrecía escolar y ii) compromiso académico. Por un lado, un alto nivel de pertenencia al colegio puede estar mostrando que los alumnos adhieren a políticas generales de cuidado en salud, las que encontramos generalmente en los establecimientos educacionales. Por otro lado, la asociación con el compromiso académico es concordante con lo encontrado en relación al rendimiento académico. Estos factores escolares parecen muy relevantes si consideramos que se ha demostrado la eficacia de intervenciones tendientes a mejorar el involucramiento de alumnos con el colegio en la prevención de conductas de riesgo $^{11}$.

Una limitación importante de este trabajo se refiere al hecho que estemos ante un estudio transversal, por lo que asociaciones causales son difíciles de inferir. Otra de las limitaciones se refiere a que otras variables no medidas pudieran ser determinantes de estas conductas. Por ejemplo, no se midieron conductas promotoras de salud en los padres ni de los profesores, las que, a través de un aprendizaje por modelamiento, pudieran influir en las conductas de los alumnos. Además, no se puede determinar con precisión los factores mediadores de algunas de las asociaciones encontradas, como es el caso de la asociación con conformidad con la apariencia física, la cual podría ser mediada por la autoestima, pero ésta no fue medida. Adicionalmente, la muestra sólo podría ser representativa de adolescentes entre $5^{\circ}$ y $8^{\circ}$ Básicos, por lo que no podemos extrapolar estos resultados de distribución y factores asociados a adolescentes mayores. Por otro lado, al ser una muestra acotada y representativa de la zona central de Chile, es posible que hayan diferencias en otras regiones del país, ya que la cultura parece afectar estas conductas ${ }^{17}$. Sería interesante observar cómo se da en otras regiones del país. Finalmente, algunos de los instrumentos están en la etapa de validación y envío para publicación, por lo que existe aún poca experiencia de su uso en Chile.

A futuro, se debe demostrar que mejorando los niveles de conductas promotoras de salud y manteniéndolos en el tiempo se pueden reducir ECNTs y otras conductas como el consumo de sustancias de abuso, violencia y embarazo adolescente. También planteamos como necesario identificar con mayor certeza qué intervenciones se pueden aplicar para mantener o modificar las conductas promotoras de salud evaluando su efectividad.

Agradecimientos: Agradecemos a todos los participantes de este estudio, en particular a los alumnos, apoderados de los colegios y a los directivos de estos establecimientos. También agradecemos a los ayudantes de investigación, en especial a Cristha Zúñiga alumna de la Escuela de Enfermería de la Universidad Valparaíso, Campus San Felipe. 


\section{Referencias}

1. World Health Organization. Milestones in Health Promotion Statements from Global Conferences. Geneva, Switzerland: World Health Organization; 2009.

2. Marriner A, Raille M. Modelos y teorías en enfermería: Elsevier Science; 2003.

3. Pender N. Health Promotion in Nursing Practice. Pender N, Murdaugh C, Parsons M, editors: Prentice Hall; 2010.

4. Sanci LA, Coffey CM, Veit FC, Carr-Gregg M, Patton GC, Day N, et al. Evaluation of the effectiveness of an educational intervention for general practitioners in adolescent health care: randomised controlled trial. BMJ 2000; 320 (7229): 224-30.

5. Wainwright $\mathrm{P}$, Thomas J, Jones $\mathrm{M}$. Health promotion and the role of the school nurse: a systematic review. J Adv Nurs 2000; 32 (5): 1083-91.

6. Sells CW, Blum RW. Morbidity and mortality among US adolescents: An overview of data and trends. Am J Public Health 1996; 86 (4): 513-9.

7. Ministerio de Interior y Seguridad Pública. Noveno Estudio Nacional de Drogas en Población Escolar de Chile, 2011. Santiago, Chile: Ministerio de Interior y Seguridad Pública; 2012.

8. Barroilhet S, Fritsch R, Guajardo V, Martínez V, Vohringer $\mathrm{P}$, Araya R, et al. [Suicidal ideation, self-directed violence and depression among Chilean school adolescents]. Rev Med Chile 2012; 140 (7): 873-81.

9. Ministerio de Salud. Encuesta Nacional de Salud ENS Chile 2009-2010. Santiago: Ministerio de Salud; 2010.

10. Ministerio de Salud. Programa Nacional de Salud Integral de Adolescentes y Jóvenes. Santiago: Ministerio de Salud; 2013.

11. Bonell C, Fletcher A, McCambridge J. Improving school ethos may reduce substance misuse and teenage pregnancy. BMJ 2007; 334 (7594): 614-6.

12. Bonell C, Parry W, Wells H, Jamal F, Fletcher A, Harden A, et al. The effects of the school environment on student health: A systematic review of multi-level studies. Health Place 2013; 21: 180-91.

13. Walker SN, Sechrist KR, Pender NJ. The Health-Promoting Lifestyle Profile: development and psychometric characteristics. Nurs Res 1987; 36 (2): 76-81.

14. Gillis AJ. The Adolescent Lifestyle Questionnaire: development and psychometric testing. Can J Nurs Res 1997 Spring; 29 (1): 29-46.

15. Hendricks C, Murdaugh C, Pender N. The Adolescent Lifestyle Profile: development and psychometric cha- racteristics. J Natl Black Nurses Assoc 2006; 17 (2): 1-5.

16. Chen MY, Wang EK, Yang RJ, Liou YM. Adolescent health promotion scale: development and psychometric testing. Public Health Nurs 2003; 20 (2): 104-10.

17. Chen MY, James K, Wang EK. Comparison of healthpromoting behavior between Taiwanese and American adolescents: a cross-sectional questionnaire survey. Int J Nurs Stud 2007; 44 (1): 59-69.

18. Hsiao YC, Chen MY, Gau YM, Hung LL, Chang SH, Tsai HM. Short-term effects of a health promotion course for Taiwanese nursing students. Public Health Nurs 2005; 22 (1): 74-81.

19. Chen MY, Chou CC, Hsu CY. The experiences of overweight female adolescents after health promotion counseling. J Nurs Res 2005; 13 (1): 41-8.

20. Blum RW, Geer L, Hutton L, McKay C, Resnick MD, Rosenwinkel K, et al. The Minnesota Adolescent Health Survey. Implications for physicians. Minn Med 1988; 71 (3): 143-5, 9 .

21. Florenzano R, Pino P, Marchandon A. [Risk behavior in adolescent students in Santiago de Chile]. Rev Med Chile 1993; 121 (4): 462-9.

22. Mihalic SF, Fagan AA, Argamaso S. Implementing the LifeSkills Training drug prevention program: factors related to implementation fidelity. Implement Sci 2008; 3: 5 .

23. Goodenow C. The psychological sense of school membership among adolescents: scale development and educational correlates. Psychology in the Schools 1993; 30 (1): 79-90.

24. Hawkins D, Guo JG, Hill K, Battin-Pearson S, Abbott R. Long-Term Effects of the Seattle Social Development Intervention on School Bonding Trajectories. Appl Dev Sci 2001; 5 (4): 225-36.

25. Srof BJ, Velsor-Friedrich B. Health Promotion in Adolescents: A Review of Pender's Health Promotion Model. Nursing Science Quarterly 2006; 19 (4): 366-73.

26. Cid P, Merino JM, Stiepovich J. [Biological and psychosocial factors that predict health promoting lifestyles]. Rev Med Chile 2006; 134 (12): 1491-9.

27. García Ph. D AW, Pender Ph. D RNNJ, Antonakos Ph. D CL, Ronis Ph. D DL. Changes in Physical Activity Beliefs and Behaviors of Boys and Girls Across the Transition to Junior High School. Journal of Adolescent Health 1998; 22 (5): 394-402.

28. Bandura A. The Primacy of Self-Regulation in Health Promotion. Applied Psychology 2005; 54 (2): 245-54. 